\title{
Pathogenesis of Atrial Fibrillation in Patients with Diabetes Mellitus: Impact on Ablation and Disease Recurrence
}

\author{
Bingxin Xie, ${ }^{1}$ Zhaojia Wang, ${ }^{1}$ Ishan Lakhani, ${ }^{2}$ Gary Tse ${ }^{1,2,3,4}$ and Tong Liu'
}

1. Tianjin Key Laboratory of Ionic-Molecular Function of Cardiovascular Disease, Department of Cardiology, Tianjin Institute of Cardiology, Second Hospital of Tianjin Medical University, Tianjin, China; 2. Cardiovascular Analytics Group, Laboratory of Cardiovascular Physiology, Hong Kong, China; 3. Faculty of Health and Medical Sciences, University of Surrey, Guildford, UK; 4. Kent and Medway Medical School, Canterbury, Kent, UK

A trial fibrillation (AF) is the most common cardiac arrhythmia observed in clinical practice worldwide, and its occurrence is reportedly higher in patients with diabetes mellitus (DM) relative to that in the general population. The underlying mechanisms governing the pathogenesis of AF in DM have not yet been completely elucidated, primarily revolving around adverse atrial structural and electrical remodelling, as well as cardiac autonomic dysfunction. These aberrations can be attributed to hyperglycaemia-induced proinflammatory changes, increased oxidative stress, and corresponding alterations in the function or expression of cardiac ion channels central to conduction. Catheter ablation is an effective treatment modality for the restoration of sinus rhythm. Prior clinical studies have examined the effectiveness and safety of catheter ablation for AF in patients with DM, but the findings have been conflicting. In addition to this, certain investigations have also attempted to risk stratify patients eligible for ablative therapy, specifically in relation to which subjects are most likely to have an improved prognosis secondary to catheter ablation. Several parameters, such as corrected QT interval, glycated haemoglobin (HDA1C) and fasting blood sugar levels, as well as left atrial diameter, have all been reported to effectively predict AF recurrence post-ablation in patients with DM.

\section{Keywords}

Atrial fibrillation, diabetes mellitus, pathogenesis, structure remodelling, electrical remodelling, catheter ablation, recurrence, predictors, QTC, HbA1C, fasting blood glucose

Disclosures: Bingxin Xie, Zhaojia Wang, Ishan Lakhani, Gary Tse and Tong Liu have no financial or non-financial relationships or activities to declare in relation to this article.

Acknowledgements: This study was supported by grants from the National Natural Science Foundation of China (81970270, 81570298 to T.L.). Review process: Double-blind peer review

Compliance with ethics: This study involves a review of the literature and did not involve any studies with human or animal subjects performed by any of the authors. Authorship: All named authors meet the criteria of the International Committee of Medical Journal Editors for authorship for this manuscript, take responsibility for the integrity of the work as a whole and have given final approval for the version to be published.

Access: This article is freely accessible at touchCARDIO.com (C) Touch Medical Media 2021.

Received: 16 September 2020

Accepted: 14 June 2021

Published online: 5 August 2021

Citation: European Journal of Arrhythmia \& Electrophysiology. 2021;7(1):26-32

Corresponding author: Tong Liu, Tianjin Key Laboratory of Ionic-Molecular Function of Cardiovascular Disease, Department of Cardiology, Tianjin Institute of Cardiology, Second Hospital of Tianjin Medical University, No. 23, Pingiiang Road, Hexi District, Tianjin 300211, China. E: liutongdoc@126.com
Atrial fibrillation (AF) is the most common cardiac arrhythmia observed in clinical practice worldwide. The incidence of $\mathrm{AF}$ is around $0.1 \%$ in individuals under 55 years old and $9 \%$ in those aged over $80 .{ }^{2}$ The global prevalence of AF is estimated to be around 44 million, which is approximately $0.5 \%$ of the total world population. ${ }^{3.4}$ Therefore, AF represents a significant public healthcare burden. Moreover, several parameters can increase the susceptibility of AF, such as ageing, hypertension and diabetes mellitus (DM). . $^{5-7}$ In a meta-analysis conducted in 2018, patients with DM showed a $49 \%$ higher risk of AF compared with the general population, and with adjusting for the risk factors of hypertension, obesity and heart disease, the relative risk was $23 \%{ }^{8}$ In addition, the incidence of various adverse outcomes is markedly increased in patients with DM and AF, compared with the general population. In 2017, a cohort study from ORBIT-AF (Outcomes Registry for Better Informed Treatment of Atrial Fibrillation) showed that patients with AF and DM had a lower quality of life, and higher risk of both all-cause and cardiovascular mortality, sudden death, as well as cardiogenic and non-cardiac hospitalization compared with patients with AF free of DM.9.10

With an increasing burden of DM in recent years, its occurrence with comorbid AF represents a unique challenge. Patients with DM and AF have several adverse outcomes, but effective treatment strategies and prevention measures remain unclear. Rhythm control, along with restoration and maintenance of sinus rhythm are important for preventing cardiovascular complications. Previous animal studies have shown that DM can reduce the effects of anti-arrhythmic drugs, but there is currently a lack of definitive clinical trial data regarding their efficacy in humans. ${ }^{11}$ Catheter ablation is a non-pharmacological approach that can be applied

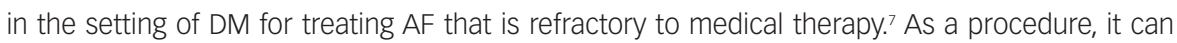
significantly improve quality of life, with data supporting its superiority relative to anti-arrhythmic therapy for long-term prognostic outcomes. ${ }^{12,13}$

The purpose of this narrative review is to summarize the underlying mechanisms governing the pathogenesis of $\mathrm{AF}$ in $\mathrm{DM}$, discuss the benefits of catheter ablation in treating patients with $\mathrm{DM}$ and $\mathrm{AF}$, detail potential clinical predictors of $\mathrm{AF}$ recurrence following catheter ablation, and illustrate possible areas of further study of AF within the context of DM. 
Figure 1: Pathophysiological mechanisms of diabetes-related atrial fibrillation

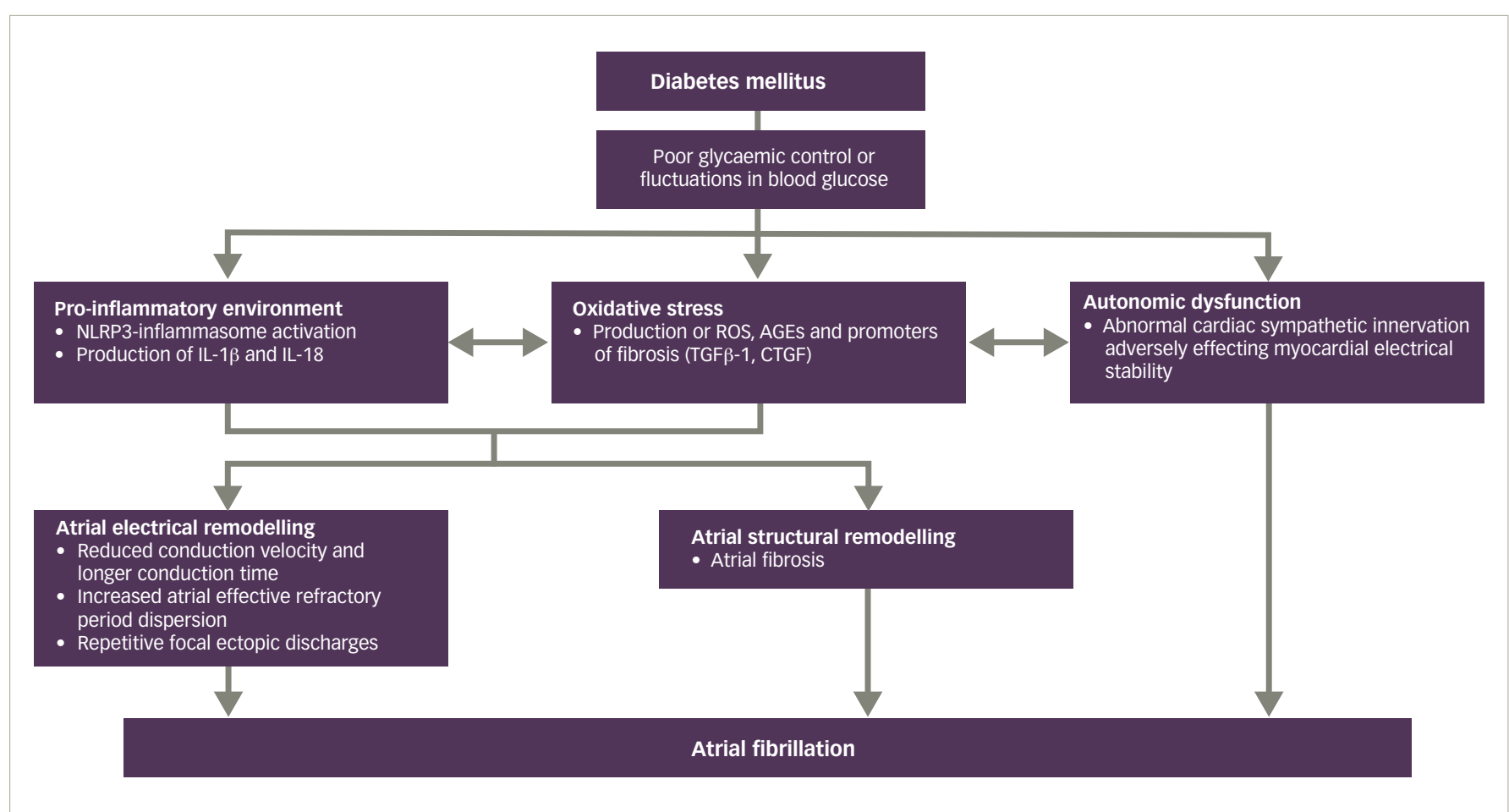

AGES = advanced glycation end products; CTGF = connective tissue growth factor; $I L=$ interleukin; NLRP3 = NACHT-LRR and PYD domain containing protein 3; ROS = reactive oxygen species; TGF $\beta-1=$ transforming growth factor beta 1.

\section{Pathogenesis of atrial fibrillation in diabetes mellitus}

The pathogenesis of AF in DM is complex (Figure 1), involving an intricate interplay of electrical and mechanical factors that coordinate in tandem to induce arrhythmia. Many of the underlying mechanisms that contribute to the development of AF in type 1 (T1DM) and type 2 DM (T2DM) are similar, as these adverse pathways tend to be initiated by components common to both phenotypes, namely glucose intolerance, a pro-inflammatory environment and oxidative stress. ${ }^{14}$ Much of the available evidence suggests that these mechanisms primarily revolve around atrial structural and electrical remodelling, as well as autonomic dysfunction. ${ }^{14-16}$ From a macroscopic perspective, structural abnormalities typically initially manifest as subendocardial interstitial fibrosis, which is subsequently reflected at the organ level as gross chamber enlargement, including left atrial dilatation and ventricular hypertrophy. ${ }^{17,18}$ Such aberrations in myocardial structure adversely impact electrical function and enhance the risk of $\mathrm{AF}$ in patients with $\mathrm{DM} .{ }^{19-23}$

In one study comparing patients with and without DM, a high proportion of patients with DM had complex fractionated atrial electrograms (CFAES) on more than $25 \%$ of their native atrial area, compared with patients without DM. ${ }^{24}$ CFAEs represent localized regions of the atria marked by reduced conduction velocity, as well as the presence of re-entry pivot points, both of which potentially stem from underlying fibrotic activity. ${ }^{25-27}$ Identification of CFAEs has implications for treatment, as they have shown to be promising targets for ablation. Current evidence indicates that pulmonary vein isolation in combination with CFAE ablation is superior in minimizing AF recurrence relative to either strategy used singularly. ${ }^{28}$ Many of the aberrations observed in diabetic hearts have also been studied on a microscopic level, providing insight into the many molecular pathways that work together to manifest the AF phenotype. The current school of thought is that the enhanced cardiac fibrosis and conduction disturbances that are typically observed in diabetic hearts chiefly occur secondary to many factors, including, but not limited to, inflammation and enhanced oxidative stress. ${ }^{29-32}$

\section{Inflammation, oxidative stress and other factors in diabetes mellitus}

First and foremost, inflammation has an important role in mediating cardiac fibrosis and subsequent electrical abnormalities in DM. ${ }^{29-32}$ There is much evidence to support the presence of an underlying chronic systemic inflammatory environment in patients with $D M{ }^{33}$ Data from a recent rabbit model revealed enhanced activity of the NACHT-LRR and PYD domain containing protein 3 (NLRP)-inflammasome in DM cardiomyocytes, which has been implicated in the development of both cardiovascular atherosclerotic disease and structural remodelling through left atrial fibrosis. ${ }^{34,35}$ Specifically, increased NLRP3-inflammasome activation in DM not only leads to the release of inflammatory cytokines that adversely affect the myocardium, namely interleukin (IL)-1 $\beta$ and IL-18, but also independently facilitates the creation of a re-entry substrate to maintain AF by shortening the atrial refractory period and inducing ectopic activity. ${ }^{34}$ Moreover, the importance of this pathway in AF development has also been showcased in studies focusing on the results of reduced NLRP3-inflammasome activity on the atria. These investigations lend further credence to the pathological role of diabetic inflammation in AF pathogenesis by demonstrating that inhibition of the NLRP3-inflammasome by glibenclamide modulates atrial structural remodelling, and reduces both conduction heterogeneity and overall AF inducibility. ${ }^{34}$

Another factor central to the development of atrial abnormalities implicated in $\mathrm{AF}$ is oxidative stress. ${ }^{29-32} \mathrm{DM}$ induces upregulation of reactive oxygen species (ROS), which, in turn, stimulates the synthesis of collagen by cardiac fibroblasts, thereby leading to fibrosis..$^{30,36-38}$ This ROS production is increased when patients with DM have either poor control of, or fluctuations in, blood glucose levels. ${ }^{39,40}$ The phenomenon of oxidative stress itself also facilitates the release of various atrial remodelling-related 
proteins that drive the progression of myocardial fibrosis, including transforming growth factor (TGF) $\beta$ - 1 , a well-defined promoter of fibrosis that mediates cellular hypertrophy, extracellular matrix (ECM) synthesis and consequent structural remodelling. ${ }^{41-45}$ In addition to this, advanced glycation end products (AGES) formed in the hyperglycaemic environment also serve as significant sources of ROS. AGEs binding to their receptors initiates signalling cascades that not only drive atrial fibrosis by enhancing the expression of connective tissue growth factor, but also aggravate myocardial inflammation and the subsequent development of diabetic cardiomyopathy by interacting with myeloid differentiation factor $2.46,47$

In addition to the increased NLRP3-inflammasome activity and ROS production that serve as a cornerstone for the initiation, maintenance and subsequent progression of AF in DM, the existing theories also propose the role of various other factors in the pathogenesis of $A F$ in DM. ${ }^{35}$ Particularly noteworthy, recent findings have illustrated that the diabetic environment activates the expression of human antigen R (HuR) in cardiac- and bone marrow-derived macrophages, which is an RNA binding protein that regulates the expression of many genes involved in inflammation, cell growth and fibrosis. ${ }^{48}$ This increased expression is accompanied by enhanced HuR nuclear-to-cytoplasmic translocation and exosomes transfer. These exosomes from macrophages, in the setting of hyperglycaemia, in turn drive cardiac fibrogenic reactions. ${ }^{46}$ Furthermore, recent investigations have also demonstrated the significance of atrial mitochondrial dysfunction in diabetic hearts. ${ }^{49-51}$ Rodent models have displayed an impairment in mitochondrial biogenesis, respiratory function and membrane depolarization in the setting of DM. These abnormalities were shown to be effectively counteracted with the use of sodium-glucose co-transporter-2 (SGLT2) inhibitor and dipeptidyl peptidase-4 (DPP-4) inhibitor drugs, which in turn may potentially have a use in the prevention of $\mathrm{AF}$ in DM. ${ }^{49-51}$

\section{Atrial electrical disturbances in diabetes mellitus}

Atrial electrical disturbances in diabetic hearts have been postulated as yet another factor that increases patient susceptibility to AF. Several investigations involving animal DM models have reported reductions in conduction velocity, lengthening of conduction time, increases in atrial effective refractory period dispersion and the existence of focal repetitive ectopic discharges, all of which collectively enhance the risk of AF. 20,42,52,53 Research using insulin-resistant rats has shown an increase in intracellular $\mathrm{Ca}^{2+}$ stores within atrial cardiomyocytes, $\mathrm{Ca}^{2+}$ transient duration and the rate of sarcoplasmic $\mathrm{Ca}^{2+}$ sparks and leakage. Further evidence of dysregulation in $\mathrm{Ca}^{2+}$ handling has been supported by the abnormal upregulation of $\mathrm{Ca}^{2+}$ homeostasis-related proteins, namely $\mathrm{Ca}^{2+} /$ calmodulin-dependent protein kinase II (CaMKII). Such aberrations in cardiomyocyte $\mathrm{Ca}^{2+}$ signalling can induce ectopic activity and $\mathrm{AF}^{54}$ Moreover, data from a recent investigation proposed the presence of circulating immunoglobulin $G$ autoantibodies within diabetic mice, which are said to in turn activate the PLC/IP3 pathway in atrial cardiomyocytes. ${ }^{52,55}$ These findings all but emphasize the central role of $\mathrm{Ca}^{2+}$ homeostatic dysregulation as a potential mechanism to explain the prevalence of AF amongst patients with DM.

It should be noted that electrical remodelling in diabetic hearts is not solely related to changes in $\mathrm{Ca}^{2+}$ signalling, but rather also to adverse alterations of cardiac ionic currents. Atrial cardiomyocytes of Zucker diabetic fatty rats have been shown to manifest with a decreased density of KV4.3 channels and corresponding transient outward $\mathrm{K}^{+}$currents $\left(\mathrm{I}_{\mathrm{to}}\right)$, as well as decreased density of KV1.5 channels and corresponding delayed rectifier $\mathrm{K}^{+}$currents $\left(\mathrm{I}_{\mathrm{Ku}}\right){ }^{20}$ These currents participate in atrial repolarization, and the consequent reduction in their expression can potentially precipitate AF. ${ }^{56,57}$ Particularly noteworthy, formerly discussed oxidative stress mechanisms can interact with and exaggerate electrical remodelling processes by promoting the downregulation of small conductance $\mathrm{Ca}^{2+}$-activated $\mathrm{K}^{+}(\mathrm{SK})$ channels in mice atria, serving as yet another substrate for arrhythmogenicity. Collectively, these ion channels are all closely related to the intrinsic electrical conduction system of the heart; as a result, subsequent disturbances in their ion transport capacities within the setting of DM likely work in a coordinated fashion to increase the risk of AF.

Furthermore, beyond the changes in transmembrane ionic transport, hyperglycaemia also modulates the expression of proteins involved in myocardial electromechanical coupling, including Connexin-43, a primary gap junction protein found through the atria and ventricles responsible for mediating cardiac conduction. ${ }^{58,59}$ Due to the central importance of such proteins to cardiac electrical homeostasis, any alterations in their synthesis or signalling induce electrical disturbances that enhance arrhythmic risk. ${ }^{60-62}$ Further evidence of such electromechanical dysfunction was shown in alloxan-induced diabetic models analysed by Doppler imaging. Relative to controls, left atrial lateral wall $\mathrm{Pa}^{\prime}$-start interval and right atrial wall Pa'-start were significantly higher in DM groups. Correlation analysis revealed an association between these parameters and subsequent cardiac fibrosis and delayed atrial electrical conduction times. ${ }^{63}$

\section{Autonomic dysfunction in diabetes mellitus}

The final area worthy of exploration relating to the pathophysiology of AF in DM is autonomic dysfunction. The general consensus regarding the role of neural remodelling in DM is somewhat unclear. Current evidence supposes that patients with DM have diminished cardiac sympathetic innervation that pathologically influences the electrical stability of the myocardium. ${ }^{64}$ More specifically, however, the diabetic phenotype is likely characterized by heterogenous sympathetic innervation, as adrenergic stimulation has been shown to enhance AF occurrence and atrial effective refractory period variability in rodent models. ${ }^{65}$ More in-depth analysis into the roles of neuronal remodelling within the setting of DM is required to fully comprehend the impact of the autonomic nervous system on AF development.

\section{Catheter ablation}

Catheter ablation has already been established as one of the effective therapies for patients with AF to restore sinus rhythm. ${ }^{66-68}$ According to the research of 521 centres from 42 countries in four continents, the efficacy of catheter ablation in the treatment of patients with either paroxysmal, persistent or long-lasting AF was about $80 \%$, and approximately $70 \%$ of patients were not required to take antiarrhythmic drug therapy (ADT) during follow-up.69 Compared with ADT, catheter ablation has demonstrated more significant clinical benefits in patients with DM. A cohort study of 70 patients with T2DM in 2009 showed that the recurrence of $\mathrm{AF}$ and the hospitalization rate was higher in the ADT group, relative to that of catheter ablation group. ${ }^{70}$

Previous cohort studies on the efficacy and safety of catheter ablation for AF in DM have demonstrated conflicting results, as summarized in Table $1^{70-79}$ The ablative approach adopted in patients with DM is largely the same as that which is taken in patients without diabetes or in those with other comorbidities. In a multicentre, prospective study from Germany, which enrolled 8,175 patients who underwent catheter ablation for AF or atrial flutter, 944 patients with DM were included. During the 1-year follow-up, there was no significant difference in arrhythmia recurrence between the patients with DM and patients without DM (46.4\% versus $46.8 \% ; p=0.90) .{ }^{72}$ In contrast, a study conducted by Chao et 
Table 1: Selected studies of safety and outcomes in patients with diabetes and atrial fibrillation ablation ${ }^{70-79}$

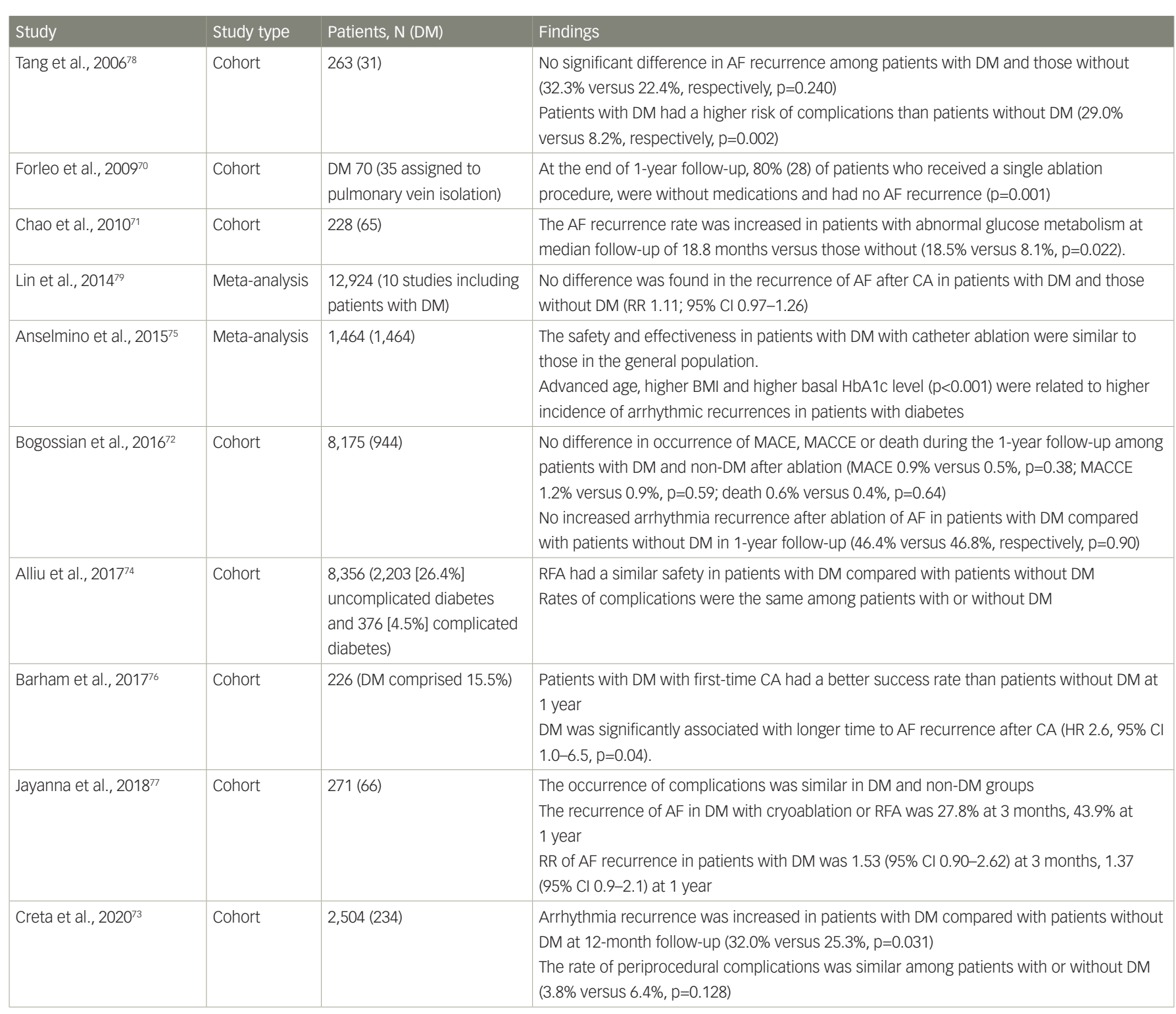

$A F=$ atrial fibrillation; $B M I=$ body mass index; $C A=$ catheter ablation; $\mathrm{Cl}=$ confidence interval; $\mathrm{DM}=$ diabetes mellitus; HbA1C = glycosylated haemoglobin; HR = hazard ratio; $M A C C E=$ major adverse cardiac and cerebrovascular events; $M A C E=$ major adverse cardiac events; $R F A=$ radiofrequency ablation; RR = relative risk.

al. showed a higher arrhythmia recurrence rate in patients with abnormal glucose metabolism. .1 This discrepancy can potentially be justified by the differences in baseline characteristics of the subjects included, whereby patients without diabetes appeared to present with a high incidence of comorbidities, including structural and coronary heart diseases, that could have possibly accounted for the similar rates of AF recurrence observed between them and their DM counterparts. ${ }^{72}$

Another investigation by creta et al. similarly demonstrated a comparatively higher rate of AF recurrence in patients with DM than in patients without DM. ${ }^{73}$ This study performed propensity-matching in order to circumvent the limitation of disparities in baseline characteristics between subjects. As such, these findings potentially support the role of aberrations in glucose tolerance in the pathophysiology of $A F$, likely through more severe alterations in atrial remodelling and conduction abnormalities in subjects with DM. ${ }^{66,68} \mathrm{In}$ an attempt to shed some light on the differences in AF incidence post-ablation demonstrated in different observational investigations, a systematic review was performed in 2015. In this meta-analysis of 1,464 patients with a follow-up of 27 months, efficacy in maintaining sinus rhythm was similar between DM and non-DM groups. ${ }^{80}$ Their meta-regression analyses demonstrated that increasing age, higher body mass index and higher baseline glycated haemoglobin (HbA1C) were associated with higher recurrence rates. These findings would suggest that catheter ablation can be effective in younger patients with DM with good glycaemic control.

Regarding procedural safety, Tang et al. reported that the rate of complications following catheter ablation for AF, such as haematomas, cardiac tamponade and stroke, was higher in subjects with DM than those without.78 However, in another larger cohort study, there was no statistically significant difference in the odds of complications for patients with DM compared to patients without DM. ${ }^{74}$ Overall, despite the findings of some studies of complications and poor outcomes in the context of DM after catheter ablation, including but not limited to stroke, thrombosis, haematoma and cardiac tamponade, the majority of the investigations examining safety outcomes of the procedure in AF treatment have not displayed any significant differences between subjects with and without DM..$^{71,78}$ 


\section{Predictors of atrial fibrillation recurrence following catheter ablation in diabetes mellitus}

The electrocardiogram (ECG) is a convenient and non-invasive diagnostic test and risk predictor. In a general population, corrected QT (QTC) interval has previously been shown to be predictive of incident $A F^{81}$ Specifically, for patients with DM undergoing catheter ablation for AF, a 2016 study (mean follow-up: $29.1 \pm 12.4$ months) reported longer QTc intervals in those with AF recurrence compared with those without $(425.2 \pm 21.5$ ms versus 414.1 $\pm 13.4 \mathrm{~ms}, \mathrm{P}=0.002$ ). For every $10 \mathrm{~ms}$ prolongation in QTC, there was a $29 \%$ higher risk of AF recurrence (hazard ratio 1.286, 95\% confidence interval 1.039-1.591, $p=0.021$ ). A cut-off of $418 \mathrm{~ms}$ showed a sensitivity of $55.7 \%$ and specificity of $69.9 \%$ for classification (area under the curve 0.65 ). ${ }^{82}$

Several alternative ECG parameters have also been investigated in relation to $A F$ recurrence post-ablation in $A F$ cohorts, albeit a certain proportion of patients did in fact present with baseline DM. Amongst assessed parameters, it has been shown that prolongation of PR interval, as well as smaller P wave duration variations in leads II and III, which refer to the difference between post- and pre-ablation $\mathrm{P}$ wave duration, are significantly associated with $\mathrm{AF}$ recurrence following multivariate adjustment..83,84 Moreover, there is also an abundance of evidence to support a correlation between ECG atrial or ventricular repolarization parameters, such as QTC dispersion, $\mathrm{P}$ wave dispersion and Tpeak-Tend interval/QTc ratio, with worsening hyperglycaemia in patients with $\mathrm{DM} .{ }^{85}$ Overall, further investigation is still required to examine the use of $\mathrm{P}$ wave duration and $\mathrm{PR}$ interval, as well as potential atrial and ventricular repolarization parameters, to predict AF recurrence because of their lack of specificity in patients with DM. Nonetheless, despite existing uncertainties, preliminary data from the studies above showcase the strong potential of ECG methods to predict AF recurrence after ablation in patients with DM within clinical settings.

Beyond ECG parameters, efficacy of blood glucose control can also influence the recurrence rate of AF after ablation. Control of glycaemic levels is crucial for DM management, and is often heterogenous within patient populations. In a notable study of 420 patients with DM who underwent AF ablation, subjects in the high fasting blood sugar group (>110 mg/dL) reportedly had an increased risk of AF recurrence compared with those with normal fasting blood sugar, thereby emphasizing the importance of adequate glycaemic control for DM prognosis. ${ }^{86}$ Another investigation, focusing instead on HbA1c levels, indicated that an elevated $\mathrm{HbA} 1 \mathrm{C}$ was predictive of post-ablation AF incidence. Specifically, a value of $\mathrm{HbA} 1 \mathrm{C} \geq 6.9 \%$ presented with a sensitivity of $55.0 \%$ and specificity of 67.4\% for AF risk prediction. ${ }^{87}$ Moreover, there are also data to suggest that patients with a worsening trend of $\mathrm{HbA} 1 \mathrm{C} 1$ year prior to ablation are more likely to develop AF following the procedure relative to patients who experienced improvements in glycaemic control during the same time period, in turn indicating that a period of optimal glucose control before ablation may have an impact on prognosis. ${ }^{88}$

Collectively, the findings of the aforementioned investigations suggest the prospective use of blood glucose control efficacy as a potential factor to assess arrhythmogenicity following catheter ablation. However, it should be noted that a possible limitation in the implementation of this parameter resides primarily in its lack of specificity to $A F$, as worsening glycaemic control has, unsurprisingly, demonstrated an association with a wide range of non-arrhythmic cardiovascular, cerebrovascular and renal outcomes as well. ${ }^{89}$

In tandem with glycaemic control, the implementation of echocardiographic parameters to examine the risk of AF recurrence after ablation has also been assessed. In a cohort of 149 patients with T2DM, left atrium size $\geq 39.0 \mathrm{~mm}$ predicted AF recurrence with $70.0 \%$ sensitivity and $55.1 \%$ specificity, even after multivariate adjustment for $\mathrm{HbA} 1 \mathrm{C}$ status and other parameters. ${ }^{88}$ Although there is a relative paucity in literature assessing the use of echocardiographic parameters to predict AF recurrence following ablation in patients with DM, many other cohort studies have illustrated the use of other variables to predict post-procedural freedom from AF, including, but not limited to, the ratio of mitral peak velocity of early filling $(E)$ to early diastolic mitral annular velocity $\left(E^{\prime}\right)$ (E/E' ratio) and left atrial appendage flow velocity. ${ }^{90}$ These findings, in turn, warrant further investigation into the use of these variables as possible markers of AF recurrence, specifically in diabetes cohorts.

\section{Potential areas for further study regarding the development of atrial fibrillation in diabetes mellitus}

Despite the abundance of literature pertaining to the relationship between DM, glycaemic control and the development of $A F$, there are several domains of this topic that warrant more investigation. ${ }^{11}$ The notion of DM as an independent predictor of AF has long since been established, and given the aforementioned mechanisms through which this relationship comes to fruition, it is not surprising to find data proposing a positive correlation between both poor glycaemic control and DM duration with AF development. ${ }^{92}$ However, there lacks sufficient research into the link between these two variables and the age of onset of AF. From a mechanistic perspective, a longer DM duration and poorer glycaemic control presumably translate into greater hyperglycaemic-induced oxidative stress and inflammatory cytokine production, which intuitively may lead to a faster rate of or more severe atrial structural and electrical remodelling that predisposes to earlier AF development.93 Although this notion may seem sound in theory, further investigation is still needed to determine the precise timing of AF onset in DM in order to establish its validity, along with its prospective influence on disease monitoring and treatment in the clinical setting.

It may also be worth assessing the likelihood of AF reversion in subjects with DM with optimal glucose control. Weight gain or obesity has been shown to be associated with suboptimal glycaemic control and poorer glucose tolerance. ${ }^{94,95}$ Data from a recent animal model has showcased that a reduction in weight is associated with a corresponding decline in inflammation, atrial fibrosis and conduction heterogeneity, leading to a reversal of atrial remodelling and AF inducibility. ${ }^{96}$ Nonetheless, whether weight reduction and, by extension, optimal glycaemic control, can themselves reduce the recurrence of $\mathrm{AF}$ in patients is as of yet uncertain, and, therefore, requires further study.

It is also abundantly clear that there is much unknown pertaining to the relative efficacy of different anti-hyperglycaemic drugs in the prevention of AF. A study examining the effects of insulin, metformin, sulfonylureas, thiazolidinediones and DPP-4 inhibitors, demonstrated that although patients on insulin were more prone to AF development, those on metformin and thiazolidinediones showed a reduction in AF incidence, whereas patients on sulfonylureas and DPP-4 inhibitors experienced no change in AF risk. ${ }^{97}$ However, despite evidence to support these findings, many other studies have not maintained this consistency. ${ }^{98-103}$ Regarding other classes of anti-hyperglycaemic drugs, glucagon-like peptide-1 receptor agonist users have also presented with varying results, as AF risk has reported to be increased with albiglutide, but unchanged with semaglutide, liraglutide and dulaglutide. ${ }^{104,105}$ In contrast, SGLT2 inhibitors have recently showed efficacy in AF prevention. ${ }^{106}$ 
With the contradictory findings concerning AF development associated with medical therapy, further study is still required in order to determine which of the available classes of anti-hyperglycaemic drugs is the most suitable option. In tandem with this, investigations have also been conducted to examine the role of such drugs in the context of catheter ablation. In a cohort of patients with DM with drug-refractory paroxysmal $A F$, treatment with pioglitazone prior to ablative therapy, significantly reduced the risk of AF recurrence. ${ }^{101}$ Similarly, the use of metformin before catheter ablation in a group of subjects with DM and AF also reportedly reduced the risk of recurrent arrhythmias. ${ }^{107}$ Despite the clear effectiveness of anti-hyperglycaemic medications in modulating disease recurrence following catheter ablation, the mechanisms through which such pharmacotherapy can prevent AF - be it directly through glycaemic control or indirectly through some of their anti-inflammatoryand anti-oxidative properties - is still unknown and necessitates greater study.

Furthermore, another pertinent point of contention in existing literature is the precise nature of the interplay between $\mathrm{DM}, \mathrm{AF}$ and incident stroke. Data from a large set of subjects with comorbid DM and AF revealed a significant association between a per unit increase in HbA1c levels and incident stroke after adjustment for $\mathrm{CHA}_{2} \mathrm{DS}_{2}$-VASC score risk factors. ${ }^{108}$ These findings are supported by those obtained from a large Danish registry, which revealed a higher risk of ischaemic stroke in patients with $\mathrm{HbA} 1 \mathrm{c}$ values of $49-58 \mathrm{mmol} / \mathrm{mol}$ and $>58 \mathrm{mmol} / \mathrm{mol}$, compared with those with values $\leq 48 \mathrm{mmol} / \mathrm{mol}$. However, it should be noted that, although this relationship remained in patients with DM for $<10$ years, it was surprisingly not maintained in patients with $D M \geq 10$ years, wherein higher HDA1C values failed to show any correlation with stroke risk. ${ }^{109}$

The importance of DM duration was further highlighted in the ATRIA California community-based cohort study, in which DM duration $\geq 3$ years was associated with an increased incidence of ischaemic stroke relative to DM duration of $<3$ years. ${ }^{110}$ Contrary to these studies, there is also some evidence to suggest an absence of any enhanced thromboembolic risk amongst patients with AF. ${ }^{111}$ Moreover, it is also necessary to contextualize such findings in relation to anticoagulant therapy for stroke prevention.

Despite current guidelines, an underuse of anticoagulant therapy in patients with $A F$ remains a persistent dilemma in healthcare. Perhaps the most important component of anticoagulant management that needs to be considered during its administration is the maintenance of a balance between preventing bleeding and thromboembolism. ${ }^{112}$ In a group derived from another nationwide Danish registry, it was revealed that longer DM duration was continuously related to a higher risk of thromboembolic events, but not to bleeding risk secondary to anticoagulant therapy, thereby possibly justifying an initiation of anticoagulation in patients with AF with prolonged DM. ${ }^{112}$ All in all, the varying reports from available investigations should incite the performance of more studies that can further clarify the precise role of glycaemic control and DM duration in stroke development secondary to AF, in order to more effectively guide the use of anticoagulant therapy in the clinical setting.

\section{Conclusions and perspectives}

In conclusion, both DM and AF have individually and collectively contributed to a significant burden on healthcare systems and economies worldwide. AF has been proven to manifest with a higher incidence in DM cohorts, and has in turn garnered greater, much warranted attention as to its pathophysiology in the setting of hyperglycaemia. The underlying mechanisms governing AF initiation and progression in DM populations is still somewhat uncertain, with factors pertaining to systemic inflammation, oxidative stress and ion channel dysfunction likely collaborating and interacting co-ordinately to adversely remodel the diabetic heart.

Catheter ablation is a widely implemented treatment method used for the recovery of sinus rhythm in patients with $A F$, including AF with DM. Whilst most studies fail to show any differences in post-procedural complication rate, there is some evidence to suggest a higher incidence of post-procedural complications amongst subjects with DM than those without DM. This, coupled with the lack of clearly defined variables, such as ECG parameters or glycaemic status, to assess for patient prognosis post-ablation, poses an additional problem towards clinical management. As such, more studies are still required to not only further elucidate the mechanism of AF susceptibility in DM, as well as the corresponding optimal therapeutic strategies for these patients, but also to provide insight on potential predictors of AF recurrence in patients with DM, particularly in relation to indices that can be obtained easily, efficiently and non-invasively. $\square$
1. Dai H, Zhang Q, Much AA, et al. Global, regional, and national prevalence, incidence, mortality, and risk factors for atrial fibrillation, 1990-2017: results from the Global Burden of Disease Study 2017. Eur Heart I Qual Care Clin Outcomes. 2020; qcaa061

2. Go AS, Hylek EM, Phillips KA, et al. Prevalence of diagnosed atrial fibrillation in adults: national implications for rhythm managentan stoke 2001:285:2370-5

3. Lippi G, Sanchis-Gomar F, Cervellin G. Global epidemiology of atrial fibrillation: An increasing epidemic and public health challenge. Int I Stroke. 2021;16:217-21.

4. Hindricks G, Potpara T, Dagres N, et al. 2020 ESC Guidelines for the diagnosis and management of atrial fibrillation developed in collaboration with the European Association for Cardio-Thoracic Surgery (EACTS): The Task Force for the diagnosis and management of atrial fibrillation of the European Society of Cardiology (ESC) Developed with the special contribution of the European Heart Rhythm Association (EHRA) of the ESC. Eur Heart J. 2021;42:373-498.

5. Kannel WB, Wolf PA, Benjamin EJ, Levy D. Prevalence, incidence, prognosis, and predisposing conditions for atrial fibrillation: prognosis, and predisposing conditions for atrial fibrillation:

Huxley RR, Alonso A, Lopez FL, et al. Type 2 diabetes, glucose Huxley RR, Alonso A, Lopez FL, et al. Type 2 diabetes, glucose
homeostasis and incident atrial fibrillation: the Atherosclerosis homeostasis and incident atrial fibrillation: the Ather
Risk in Communities study. Heart. 2012;98:133-8 Risk in Communities study. Heart. 2012;98:133-8.
Benjamin EL, Levy D, Vaziri SM, et al. Independent risk Benjamin EL, Levy D, Vaziri SM, et al. Independent risk
factors for atrial fibrillation in a population-based cohort. The Framingham Heart Study. JAMA. 1994;271:840-4.

8. Xiong Z, Liu T, Tse G, et al. A machine learning aided systematic review and meta-analysis of the relative risk of atrial fibrillation in patients with diabetes mellitus. Front Physiol. 2018;9:835.

9. Echouffo-Tcheugui JB, Shrader P, Thomas L, et al. Care patterns and outcomes in atrial fibrillation patients with and without diabetes: ORBIT-AF Registry. J Am Coll Cardiol. 2017;70:1325-35. 10. Du X, Ninomiya T, de Galan B, et al. Risks of cardiovascular events and effects of routine blood pressure lowering among patients with type 2 diabetes and atrial fibrillation: results of the ADVANCE study. Eur Heart J. 2009;30:1128-35.

11. Ito I, Hayashi Y, Kawai Y, et al. Diabetes mellitus reduces the antiarrhythmic effect of ion channel blockers. Anesth Analg. 2006:103:545-50.

12. Blomström-Lundqvist C, Gizurarson S, Schwieler 」, et al. Effect of catheter ablation vs antiarrhythmic medication on quality of
life in patients with atrial fibrillation: the CAPTAF randomized life in patients with atrial fibrillation:

3. Asad ZUA, Yousif A, Khan MS, et al. Catheter ablation versus medical therapy for atrial fibrillation: a systematic review and meta-analysis of randomized controlled trials. Circ Arrhythm Electrophysiol. 2019;12:e007414

14. Grisanti LA. Diabetes and arrhythmias: pathophysiology, mechanisms and therapeutic outcomes. Front Physio. 2018;9:1669

15. Zhang Q, Liu T, Ng CY, Li G. Diabetes mellitus and atrial remodeling: mechanisms and potential upstream therapies. Cardiovasc Ther. 2014:32:233-41.

16. Goudis CA, Korantzopoulos P, Ntalas IV, et al. Diabetes mellitus and atrial fibrillation: Pathophysiological mechanisms and potential upstream therapies. Int I Cardiol. 2015:184:617-22.

17. Tadic M, Cuspidi $C$. The influence of type 2 diabetes on left Tadic M, Cuspidi C. The influence of type 2 diab
atrial remodeling. Clin Cardiol. 2015;38:48-55.

18. Sun Y, Hu D. The link between diabetes and atrial fibrillation: cause or correlation? J Cardiovasc Dis Res. 2010;1:10-1.

19. Lu L, Ma J, Sun M, et al. Melatonin ameliorates MI-induced cardiac remodeling and apoptosis through a JNK/p53dependent mechanism in diabetes mellitus. Oxid Med Cell Longev. 2020;2020:1535201.

20. Fu L, Rao F, Lian $\mathrm{F}$ et al. Mechanism of electrical remodeling of atrial myocytes and its influence on susceptibility to atria fibrillation in diabetic rats. Life Sci. 2019;239:116903.

21. Russo I, Frangogiannis NG. Diabetes-associated cardiac fibrosis: Cellular effectors, molecular mechanisms and therapeutic opportunities. J Mol Cell Cardiol. 2016;90:84-93.

22. Tiwari S, Schirmer H, Jacobsen BK, et al. Association between diastolic dysfunction and future atrial fibrillation in the Tromsø Study from 1994 to 2010. Heart. 2015:101:1302-8.

23. De Blasio MJ, Huynh N, Deo M, et al. Defining the progression of diabetic cardiomyopathy in a mouse model of type 1 diabetes. diabetic cardiomyopathy in

24. Grieco D, Palamà Z, Borrelli A, et al. Diabetes mellitus and atrial remodelling in patients with paroxysmal atrial fibrillation: role of electroanatomical mapping and catheter ablation. Diab Vasc Dis Res. 2018;15:185-95

25. de Bakker JM, Wittkampf FH. The pathophysiologic basis of fractionated and complex electrograms and the impact of recording techniques on their detection and interpretation. Circ Arrhythm Electrophysiol. 2010;3:204-13

26. Konings KT, Smeets JL, Penn OC, et al. Configuration of unipolar atrial electrograms during electrically induced atrial fibrillation in humans. Circulation. 1997;95:1231-41.

27. Jacquemet $V$ Henriquez $C S$. Cenesis of complex fractionated Jacquemet $V$, Henriquez $\mathrm{Cs}$. Genesis of complex fractionated atria

.

28. Verma A, Mantovan R, Macle L, et al. Substrate and Trigge Ablation for Reduction of Atrial Fibrilation (STAR AF). a randomized, multicentre, international trial. Eur Heart $J$ 2010;31:1344-56

29. Korantzopoulos P, Letsas KP, Tse G, et al. Inflammation and atrial fibrillation: a comprehensive review. J Arrhythm. 2018;34:394-401

30. Bohne L, Johnson D, Rose RA, et al. The association between diabetes mellitus and atrial fibrillation: clinical and mechanistic insights. Front Physiol. 2019;10:135

31. Zhang Z, Zhang X, Meng L, et al. Pioglitazone inhibits 
diabetes-induced atrial mitochondrial oxidative stress and improves mitochondrial biogenesis, dynamics, and function through the PPAR- - PGC- $1 \alpha$ signalling pathway. Front Pharmacol. 2021:12:658362

32. Korantzopoulos $\mathrm{P}$, Letsas $\mathrm{K}$, Fragakis $\mathrm{N}$, et al. Oxidative stress and atrial fibrillation: an update. Free Radic Res. 2018;52:1199-209.

33. Duncan BB, Schmidt MI, Pankow JS, et al. Atherosclerosis risk in communities, low-grade systemic inflammation and the development of type 2 diabetes: the atherosclerosis risk in communities study. Diabetes. 2003;52:1799-805.

34. WU X, Liu Y, Tu D, et al. Role of NLRP3-inflammasome/ caspase-1/galectin-3 pathway on atrial remodeling in diabetic rabbits. J Cardiovasc Trans/ Res. 2020;13:731-40.

35. Yao C, Veleva T, Scott L Jr., et al. Enhanced cardiomyocyte NLRP3 inflammasome signaling promotes atrial fibrillation. Circulation. 2018;138:2227-42

36. Liang $X$, Zhang $Q$, Wang $X$, et al. Reactive oxygen species mediated oxidative stress links diabetes and atrial fibrillation. Mol Med Rep. 2018:17:4933-40.

37. De Blasio MJ, Huynh K, Qin C, et al. Therapeutic targeting of oxidative stress with coenzyme Q10 counteracts exaggerated diabetic cardiomyopathy in a mouse model of diabetes with diminished PI3K(p110 $\alpha$ ) signaling. Free Radic Biol Med. 2015;87:137-47.

38. Gong $\mathrm{M}$, Yuan $\mathrm{M}$, Meng $\mathrm{L}$, et al. Wenxin Keli regulates mitochondrial oxidative stress and homeostasis and improves atrial remodeling in diabetic rats. Oxid Med Cell Longev. 2020;2020:2468031.

39. Saito S, Teshima Y, Fukui A, et al. Glucose fluctuations increase the incidence of atrial fibrillation in diabetic rats. Cardiovasc Res. 2014:104:5-14.

40. Wang Q, Wang J, Wang P, et al. Glycemic control is associated with atrial structural remodeling in patients with type 2 diabetes. BMC Cardiovasc Disord. 2019:19:278.

41. Liu C, Liu R, Fu H, et al. Pioglitazone attenuates atria remodeling and vulnerability to atrial fibrillation in alloxan-induced diabetic rabbits. Cardiovasc Ther 2017;35:e12284.

42. Yang Y, Zhao J, Qiu J, et al. Xanthine oxidase inhibitor allopurino prevents oxidative stress-mediated atrial remodeling in alloxan-induced diabetes mellitus rabbits. J Am Heart ASSOC 2018;7:e008807

43. Eid RA, Alkhateeb MA, El-Kott AF, et al. A high-fat diet rich in corn oil induces cardiac fibrosis in rats by activating JAK2 STAT3 and subsequent activation of ANG $11 / T$ TGF-1 $1 / /$ Smad3 pathway: the role of ROS and IL-6 trans-signaling. J Food Biochem. 2019:43:e12952.

44. Qi Y, WU H, Mai C, et al. LncRNA-MIAT-mediated miR-214-3p silencing is responsible for IL-17 production and cardiac fibrosis in diabetic cardiomyopathy. Front Cell Dev Biol. 2020;8:243.

45. Goldfarb S, Ziyadeh FN. TGF-beta: a crucial component of the pathogenesis of diabetic nephropathy. Trans Am Clin Climato Assoc. 2001:112:27-32.

46. Govindappa PK, Patil M, Garikipati VNS, et al. Targeting exosome-associated human antigen $R$ attenuates fibrosis and inflammation in diabetic heart. FASEB J. 2020;34:2238-51.

47. Wang Y, Luo W, Han J, et al. MD2 activation by direct AGE interaction drives inflammatory diabetic cardiomyopathy. Nat Commun. 2020;11:2148.

48. Green LC, Anthony SR, Slone S, et al. Human antigen R as a therapeutic target in pathological cardiac hypertrophy. $J \mathrm{CI}$ Insight. 2019:4:e121541.

49. Faria A, Persaud SJ. Cardiac oxidative stress in diabetes: mechanisms and therapeutic potential. Pharmacol The 2017;172:50-62.

50. Shao Q, Meng L, Lee S, et al. Empagliflozin, a sodium glucose co-transporter-2 inhibitor, alleviates atrial remodeling and improves mitochondrial function in high-fat diet/streptozotocininduced diabetic rats. Cardiovasc Diabetol. 2019;18:165.

51. Zhang X, Zhang Z, Yang Y, et al. Alogliptin prevents diastol dysfunction and preserves left ventricular mitochondrial function in diabetic rabbits. Cardiovasc Diabetol. 2018;17:160.

52. Chan YH, Chang GJ, Lai YJ, et al. Atrial fibrillation and its arrhythmogenesis associated with insulin resistance. Cardiovasc Diabetol. 2019;18:125

53. Yi F, Ling TY, Lu T, et al. Down-regulation of the small conductance calcium-activated potassium channels in diabetic mouse atria. J Biol Chem. 2015;290:7016-26.

54. Heijman J, Voigt N, Nattel S, Dobrev D. Cellular and molecular electrophysiology of atrial fibrillation initiation, maintenance, and progression. Circ Res. 2014;114:1483-99.

55. Luo Y, Liu X, Ma R, et al. Circulating IgGs in type 2 diabetes with atrial fibrillation induce IP(3)-mediated calcium elevation in cardiomyocytes. iscience. 2020;23:101036.

56. Schotten U, Verheule S, Kirchhof P, Goette A. Pathophysiologica mechanisms of atrial fibrillation: a translational appraisal. Physiol Rev. 2011;91:265-325.

57. Ravens $U$, Wettwer E. Ultra-rapid delayed rectifier channels: molecular basis and therapeutic implications. Cardiovasc Res. 2011;89:776-85

58. van der Velden $\mathrm{HM}$, Jongsma HJ. Cardiac gap junctions and connexins: their role in atrial fibrillation and potential as therapeutic targets. Cardiovasc Res. 2002;54:270-9.

59. Tse G, Yeo JM. Conduction abnormalities and ventricular arrhythmogenesis: The roles of sodium channels and gap unctions. Int J Cardiol Heart VasC. 2015;9:75-82.

60. Wang GY, Bi YG, Liu XD, et al. Autophagy was involved in the protective effect of metformin on hyperglycemia-induced cardiomyocyte apoptosis and Connexin43 downregulation in H9c2 cells. Int I Med Sci. 2017:14:698-704.

61. Mitašíková $\mathrm{M}$, Lin $\mathrm{H}$, Soukup T, et al. Diabetes and thyroid hormones affect connexin-43 and PKC-epsilon expression in rat heart atria. Physiol Res. 2009;58:211-7.

62. Zhang $Y$, Wang $Y$, Yanni J, et al. Electrical conduction system remodeling in streptozotocin-induced diabetes mellitus rat heart. Front Physiol. 2019;10:826.

63. Fu H, Liu C, Li J, et al. Impaired atrial electromechanical function and atrial fibrillation promotion in alloxan-induced diabetic rabbits. Cardiol J. 2013;20:59-67.

64. Stevens MJ, Raffel DM, Allman KC, et al. Regression and progression of cardiac sympathetic dysinnervation complicating diabetes: an assessment by $\mathrm{C}-11$ hydroxyephedrine and positron emission tomography. Metabolism. 1999:48:92-101.

65. Otake $\mathrm{H}$, Suzuki $\mathrm{H}$, Honda T, Maruyama Y. Influences of autonomic nervous system on atrial arrhythmogenic substrates and the incidence of atrial fibrillation in diabetic heart. Int Heart J. 2009;50:627-41.

66. Liu T, Tse G, Yu L, et al. Atrial fibrillation: mechanisms and management. Cardiol Res Pract. 2019;2019:890937

67. Li KHC, Dong M, Gong $\mathrm{M}$, et al. International health informatics study, atrial fibrillation recurrence and peri-procedural complication rates in nMARQ vs. conventional ablation techniques: a systematic review and meta-analysis. Front Physiol. 2018;9:544.

68. Rottner L, Bellmann B, Lin T, et al. Catheter ablation of atrial fibrillation: state of the art and future perspectives. Cardiol Ther. 2020;9:45-58.

69. Cappato R, Calkins H, Chen SA, et al. Updated worldwide survey on the methods, efficacy, and safety of catheter ablation for human atrial fibrillation. Circ Arrhythm Electrophysiol. 2010;3:32-8

70. Forleo GB, Mantica M, De Luca L, et al. Catheter ablation of atrial fibrillation in patients with diabetes mellitus type 2 : results from a randomized study comparing pulmonary vein isolation versus antiarrhythmic drug therapy. I Cardiovasc Electrophysiol. 2009;20:22-8

71. Chao TF, Suenari K, Chang SL, et al. Atrial substrate properties and outcome of catheter ablation in patients with paroxysma atrial fibrillation associated with diabetes mellitus or impaired fasting glucose. Am J Cardiol. 2010;106:1615-20.

72. Bogossian H, Frommeyer G, Brachmann J, et al. Catheter ablation of atrial fibrillation and atrial flutter in patients with diabetes mellitus: who benefits and who does not? Data from the German ablation registry. Int I Cardiol. 2016;214:25-30.

73. Creta A Providência R Adragão P, et al Impact of type-2 diabetes mellitus on the outcomes of catheter ablation of atria fibrillation (European observational multicentre study). $A m$ Cardiol. 2020;125:901-6.

74. Alliu SE, Adejumo A, M Durojaiye, et al. Abstract 072: Impact of chronic diabetes on periprocedural outcomes among patient with atrial fibrillation and flutter who underwent radiofrequency catheter ablation therapy (RFA). Report from the NIS 2014. Circ Cardiovasc Qual Outcomes. 2017;10(Suppl 3).

75. Anselmino M, Matta M, D'Ascenzo F, et al. Catheter ablation of atrial fibrillation in patients with diabetes mellitus: a systematic review and meta-analysis. Europace. 2015;17:1518-25.

76. Barham W, Sauer W, Fleeman B, et al. 'Diabetes mellitus and late outcomes after atrial fibrillation catheter ablation' in Abstracts from the 2017 International Atrial Fibrillation Association. J Cardiovasc Electrophysiol. 2017:28:586-7. Abstract 14.

77. Bengaluru Jayanna M, Mohsen AM, Palamaner Subash Shantha $\mathrm{G}$, et al. Outcomes of catheter ablation of atrial fibrillation in patients with diabetes mellitus. Cardiology. 2018;140(Suppl. 1):272

78. Tang RB, Dong JZ, Liu XP, et al. Safety and efficacy of catheter ablation of atrial fibrillation in patients with diabetes mellitus--single center experience. J Interv Card Electrophysio. 2006; 17:41-6.

79. Lin KJ, Cho SI, Tiwari N, et al. Impact of metabolic syndrome on the risk of atrial fibrillation recurrence after catheter ablation: systematic review and meta-analysis. I Interv Card Electrophysiol. 2014;39:211-23.

80. Chun KRJ, Okumura K, Scazzuso F, et al. Safety and efficacy of cryoballoon ablation for the treatment of paroxysmal and persistent $\mathrm{AF}$ in a real-world global setting: results from the Cryo AF Global Registry. J Arrhythm. 2021;37:356-67.

81. Zhang N, Gong M, Tse G, et al. Prolonged corrected QT interval in predicting atrial fibrillation: a systematic review and

meta-analysis. Pacing Clin Electrophysiol. 2018;41:321-7.

Plun-Favreau H, Klupsch K, Moisoi N, et al. The mitochondrial
protease HtrA2 is regulated by Parkinson's disease-associated kinase PINK1. Nat Cell Biol. 2007;9:1243-52.

83. Wu J-T, Dong J-Z, SangC-H, et al. Prolonged PR interval and risk of recurrence of atrial fibrillation after catheter ablation. Int Heart J. 2014;55:126-30.

84. Hu X, Jiang J, Ma Y, Tang A. Novel P wave indices to predict atrial fibrillation recurrence after radiofrequency ablation for paroxysmal atrial fibrillation. Med Sci Monit. 2016;22:2616-23.

85. Kuzu F. The effect of type 2 diabetes on electrocardiographic markers of significant cardiac events. Pak J Med Sci.
2018;34:626-32.

86. Mohanty S, Asfour I, Mohanty P, et al. Baseline fasting blood sugar predicts long-term outcome of catheter ablation in atrial fibrillation. J Am Coll Cardiol. 2016:67(Suppl. 13):797.

87. Lu ZH, Liu N, Bai R, et al. HbA1c levels as predictors of ablation outcome in type 2 diabetes mellitus and paroxysmal atrial fibrillation. Herz. 2015;40(Suppl. 2):130-6

88. Donnellan E, Aagaard P, Kanj M, et al. Association between pre-ablation glycemic control and outcomes among patients with diabetes undergoing atrial fibrillation ablation. JACC Clin Electrophysiol. 2019;5:897-903

89. Kishore P, Kim SH, Crandall JP. Glycemic control and cardiovascular disease: what's a doctor to do? Curr Diab Rep. 2012;12:255-64.

90. Kim YG, Choi II, Boo KY, et al. Clinical and echocardiographic risk factors predict late recurrence after radiofrequency catheter ablation of atrial fibrillation. Sci Rep. 2019;9:6890.

91. Aune D, Feng T, Schlesinger S, et al. Diabetes mellitus, blood glucose and the risk of atrial fibrillation: a systematic review and meta-analysis of cohort studies. I Diabetes Complications. 2018;32:501-11.

92. Wang A, Green JB, Halperin JL, Piccini JP Sr. Atrial fibrillation and diabetes mellitus: JACC review topic of the week. J Am Coll Cardiol. 2019;74:1107-15.

93. Oguntibeju OO. Type 2 diabetes mellitus, oxidative stress and inflammation: examining the links. Int J Physiol Pathophysiol Pharmacol. 2019;11:45-63.

94. Bae JP, Lage MJ, Mo D, et al. Obesity and glycemic control in patients with diabetes mellitus: analysis of physician electronic health records in the US from 2009-2011. J Diabetes Complications. 2016;30:212-20.

95. Ferrannini E, Camastra S. Relationship between impaired glucose tolerance, non-insulin-dependent diabetes mellitus and obesity. Eur I Clin Invest. 1998:28(Suppl. 2):3-6; discussion 6-7.

96. Mahajan R, Lau DH, Brooks AG, et al. Atrial fibrillation and obesity: reverse remodeling of atrial substrate with weight reduction. JACC Clin Electrophysiol. 2021;7:630-41.

97. Liou YS, Yang FY, Chen HY, Jong GP. Antihyperglycemic drugs use and new-onset atrial fibrillation: a population-based nested case control study. PLOS One. 2018;13:e0197245.

98. Pallisgaard JL, Lindhardt TB, Staerk L, et al. Thiazolidinediones are associated with a decreased risk of atrial fibrillation compared with other antidiabetic treatment: a nationwide cohort study. Eur Heart J Cardiovasc Pharmacother. 2017;3:140-6.

99. Chang SH, WU LS, Chiou MJ, et al. Association of metformin with lower atrial fibrillation risk among patients with type 2 diabetes mellitus: a population-based dynamic cohort and in vitro studies. Cardiovasc Diabetol. 2014;13:123.

100. Chao TF, Leu HB, Huang CC, et al. Thiazolidinediones can prevent new onset atrial fibrillation in patients with non-insulin dependent diabetes Int I Cardiol. 2012:156:199-202.

101. Gu J, Liu X, Wang X, et al. Beneficial effect of pioglitazone on the outcome of catheter ablation in patients with paroxysma atrial fibrillation and type 2 diabetes mellitus. Europace. 2011;13:1256-61

102. Chang $\mathrm{CY}$, Yeh $\mathrm{YH}$, Chan YH, et al. Dipeptidyl peptidase-4 inhibitor decreases the risk of atrial fibrillation in patients with type 2 diabetes: a nationwide cohort study in Taiwan. Cardiovasc Diabetol. 2017;16:159.

103. Dormandy JA, Charbonnel B, Eckland DJ, et al. Secondary prevention of macrovascular events in patients with type 2 diabetes in the PROactive Study (PROspective pioglitAzone Clinical Trial In macrovascular Events): a randomised controlled trial. Lancet. 2005:366:1279-89.

104. Fisher M, Petrie MC, Ambery PD, et al. Cardiovascular safety of albiglutide in the Harmony programme: a meta-analysis. Lancet Diabetes Endocrinol. 2015:3:697-703.

105. Lăcătușu CM, Grigorescu E-D, Stătescu C, et al. Association of antihyperglycemic therapy with risk of atrial fibrillation and stroke in diabetic patients. Medicina (Kaunas). 2019:55:592.

106. Li D, Liu Y, Hidru TH, et al. Protective effects of sodium-glucose transporter 2 inhibitors on atrial fibrillation and atrial flutter: a systematic review and meta-analysis of randomized placebocontrolled trials. Front Endocrinol (Lausanne). 2021;12:619586.

107. Deshmukh A, Ghannam M, Liang J, et al. Effect of metformin on outcomes of catheter ablation for atrial fibrillation. $J$ Cardiovasc Electrophysiol. 2021;32:1232-9.

108. Saliba W, Barnett-Griness O, Elias M, Rennert G. Glycated hemoglobin and risk of first episode stroke in diabetic patients with atrial fibrillation: a cohort study, Heart Rhythm. 2015;12:886-92

109. Fangel MV Nielsen PB, Kristensen JK, et al. Glycemic status and thromboembolic risk in patients with atrial fibrillation and type 2 diabetes mellitus: a Danish cohort study Circ Arrhythm Electrophysiol. 2019;12:e007030

110. Ashburner JM, Go AS, Chang Y, et al. Effect of diabetes and glycemic control on ischemic stroke risk in AF patients: ATRIA study. J Am Coll Cardiol. 2016;67239-47.

111. Patti $G$, Lucerna $M$, Cavallari I, et al. Insulin-requiring versus noninsulin-requiring diabetes and thromboembolic risk in patients with atrial fibrillation: PREFER in AF. J Am Coll Cardiol. 2017;69:409-19

112. Overvad TF, Skjoth F, Lip GY, et al. Duration of diabetes mellitus and risk of thromboembolism and bleeding in atrial fibrillation: nationwide cohort study. Stroke. 2015;46:2168-74. 